\title{
Fast and selective phonon-assisted state preparation of a quantum dot by adiabatic undressing
}

\author{
A.M. Barth ${ }^{1}$, S. Lüker ${ }^{2}$, A. Vagov ${ }^{1}$, D.E. Reiter ${ }^{2,3}$, T. Kuhn ${ }^{2}$ and V.M. Axt ${ }^{1}$ \\ ${ }^{1}$ Institut für Theoretische Physik III, Universität Bayreuth, 95440 Bayreuth, Germany * \\ ${ }^{2}$ Institut für Festkörpertheorie, Universität Münster, 48149 Münster, Germany and \\ ${ }^{3}$ Imperial College London, South Kensington Campus, London SW7 2AZ, UK.
}

(Dated: April 12, 2018)

\begin{abstract}
We investigate theoretically the temporal behavior of a quantum dot under off-resonant optical excitation targeted at fast acoustic phonon-assisted state preparation. We demonstrate that in a preparation process driven by short laser pulses three processes can be identified: a dressing of the states during the switch on of the laser pulse, a subsequent phonon-induced relaxation and an undressing at the end of the pulse. By analyzing excitation scenarios with different pulse shapes we highlight the decisive impact of an adiabatic undressing on the final state in short pulse protocols. Furthermore, we show that in exciton-biexciton systems the laser characteristics such as the pulse detuning and the pulse length as well as the biexciton binding energy can be used to select the targeted quantum dot state.
\end{abstract}

\section{INTRODUCTION}

Many of today's proposals for quantum information applications $\underline{\underline{1}}$ rely on the controlled and fast manipulation of the discrete states of the corresponding devices' underlying structures. Semiconductor quantum dots (QDs) are frequently discussed as building blocks for such materials because they hold out the prospect of tailor-made energy spectra and a high integrability in a solid-state environment. The excitonic QD states are promising candidates to be used as qubits for quantum computing ${ }^{2-\underline{7}}$, while the radiative decay from the biexciton cascade offers the possibility of an on-demand creation of indistinguishable entangled photon pairs ${ }^{8-10}$.

It has been shown that both exciton and biexciton states of a QD can be prepared by using ultra-fast laser pulses under a variety of excitation conditions 11,12 . The most commonly known schemes for this purpose are resonant excitation leading to Rabi rotations $\underline{13}-16$, different protocols using chirped laser pulses exploiting the adiabatic rapid passage effect $17-25$, and phonon-assisted off-resonant driving 26-29. Recently, the latter method has also been experimentally demonstrated ${ }^{30}-32$. Indeed, there is an increased interest in this approach, because it is not only stable against fluctuations of the applied field intensity, but also leaves the quantum dot transition laser-free, which can be important when the emitted photons need to be spectrally separated from the laser pulse. Furthermore, in contrast to the other two protocols the phonon-assisted scheme makes active use of the phonon coupling and even works the better the stronger this coupling is.

In this paper, we examine the influence of the pulse shape on the phonon-assisted state preparation. We identify three distinct processes that take place during the laser-driven evolution of the QD states. When the pulse starts the coupling to the laser-field leads to a dressing of the bare QD states. This enables a phonon-induced relaxation between the dressed states $26,33,34$ and finally when the pulse is switched off an undressing takes place. While previous studies mostly concentrated on the phonon induced relaxation and the resulting exciton and biexciton occupation obtained when applying an off-resonant driving pulse ${ }^{29}$, here we will explain the impact of all three different processes and examine in detail the role of the switch-on and switch-off phase of the excitation. We will show that within the relaxation process there is a tradeoff situation between a sufficiently fast preparation and an optimal preparation fidelity and that for a high fidelity preparation of the bare QD states an adiabatic undressing, that can be realized by a long enough switch-off time, is indispensable in short pulse protocols. Our analysis also shows that even though Gaussian pulses, as used in very recent experiments $30-32$, are not the ideal choice for the phonon-assisted protocol they fulfill the requirements for fast state preparation surprisingly well for a wide intensity range provided that the regime of non-adiabatic dynamics is not yet reached. The latter condition sets a lower bound to the pulse duration.

The pulse characteristics not only allow to control the fidelity of the achieved inversion, but in addition can be used to select the QD state that is targeted by the phonon-assisted process. For the two-level case the sign of the pulse detuning determines whether the QD is driven towards the ground-state or the exciton state. For the exciton-biexciton system also the biexciton binding energy and the pulse length play a critical role in determining the targeted QD state. Understanding that the preparation is a three-step process gives us an intuitive answer to the important question which state is selected by the phonon-assisted preparation scheme.

\section{MODEL}

We consider a strongly confined GaAs QD driven by an external laser field and coupled to a continuum of acoustic bulk phonons. Our model is defined by the Hamiltonian $H=H_{\text {dot, light }}+H_{\text {dot-ph }}$, i.e., the electronic system coupled to the laser field and an additional phonon part. 
Let us first concentrate on

$$
H_{\mathrm{dot}, \text { light }}=\sum_{\nu} \hbar \omega_{\nu}|\nu\rangle\left\langle\nu\left|+\sum_{\nu \nu^{\prime}} \hbar M_{\nu \nu^{\prime}}\right| \nu\right\rangle\left\langle\nu^{\prime}\right|,
$$

where $|\nu\rangle$ are the electronic basis states with corresponding energies $\hbar \omega_{\nu}$. The matrix element $M_{\nu \nu^{\prime}}$ describes the coupling between the QD and the classical laser field using the common dipole and rotating wave approximations. In the first part of the paper we will restrict ourselves to a two-level system consisting of the ground-state $|0\rangle$, for which we set $\hbar \omega_{0}=0$, and a single exciton state $|X\rangle$ with energy $\hbar \omega_{X}$ as illustrated in Fig. 1(a). The two-level approximation is valid when considering circularly polarized light with a single polarization orientation and the exchange interaction is negligibly small. The exchange interaction strongly depends on the QD geometry ${ }^{35}$ and can be close to zero, as it is favorable for, e.g., entangled photon creation ${ }^{9}$. In this case the non-zero matrix elements of the light-matter coupling are given by

$$
M_{0 X}=\frac{1}{2} f(t) e^{i \omega_{L} t}, \quad M_{X 0}=M_{0 X}^{*},
$$

where $f(t)$ is a real pulse envelope function, which in the following is referred to as field strength. The field strength $f(t)$ is related to the electric field $\mathbf{E}(t)$ with frequency $\omega_{L}$ and the QD dipole matrix element $\mathbf{d}$ by $-\mathbf{d} \cdot \mathbf{E}(t)=\frac{\hbar}{2} f(t) e^{-i \omega_{L} t}$.

A very useful picture for strongly driven few-level systems, which we will employ to analyze our results, is the dressed state picture. The dressed states are the eigenstates of the coupled light-matter Hamiltonian in a frame rotating with the laser frequency $\stackrel{36}{ }$. For the two-level system driven by a laser-field with a fixed field strength $f$ and a detuning $\Delta$ they are given by the expressions

$$
\begin{aligned}
\left|\psi_{\text {up }}\right\rangle & =+\cos (\Theta)|0\rangle+\sin (\Theta)|X\rangle \\
\left|\psi_{\text {low }}\right\rangle & =-\sin (\Theta)|0\rangle+\cos (\Theta)|X\rangle
\end{aligned}
$$

where $\Theta$ is the mixing angle defined by $\tan (\Theta)=\frac{\hbar f}{\Delta+\hbar \Omega}$ and $\Omega$ is the Rabi frequency given by

$$
\hbar \Omega=\sqrt{(\hbar f)^{2}+\Delta^{2}} .
$$

The corresponding dressed state energies read

$$
E_{\text {up } / \text { low }}=\frac{1}{2}(-\Delta \pm \hbar \Omega) .
$$

It is worth noting that the contributions of the ground and exciton states to the dressed states vary depending on the detuning and the field strength.

The final part of this paper will be devoted to excitations with linearly polarized light. In this case one also has to take into account the biexciton state and the system described so far needs to be extended to a three-level model consisting of the ground state $|0\rangle$, the single exciton state $|X\rangle$ and the biexciton state $|B\rangle$. Note that the single exciton state $|X\rangle$ in the three-level system has different polarization than in the two-level system described further above. As is illustrated in Fig. [1(b), the biexciton state has the energy $\hbar \omega_{B}=2 \hbar \omega_{X}-\Delta_{B}$, where $\Delta_{B}$ is the biexciton binding energy. In the exciton-biexciton system, the non-vanishing dipole matrix elements are given by

$$
M_{0 X}=M_{X B}=\frac{1}{2} f(t) e^{i \omega_{L} t}, M_{X 0}=M_{B X}=M_{0 X}^{*} .
$$

Let us now focus on the coupling of the QD to the phonon environment. We model the electron-phonon interaction by a pure-dephasing Hamiltonian which, together with the free phonon Hamiltonian, has the form

$$
H_{\mathrm{dot}-\mathrm{ph}}=\sum_{\mathbf{q}} \hbar \omega_{\mathbf{q}} b_{\mathbf{q}}^{\dagger} b_{\mathbf{q}}+\sum_{\mathbf{q} \nu} \hbar n_{\nu}\left(\gamma_{\mathbf{q}} b_{\mathbf{q}}+\gamma_{\mathbf{q}}^{*} b_{\mathbf{q}}^{\dagger}\right)|\nu\rangle\langle\nu| .
$$

The operators $b_{\mathbf{q}}^{\dagger}\left(b_{\mathbf{q}}\right)$ create (annihilate) a phonon with wave vector $\mathbf{q}$ and linear dispersion $\hbar \omega_{\mathbf{q}}=\hbar c_{s}|\mathbf{q}|$, where $c_{s}$ denotes the longitudinal sound velocity. $n_{\nu}$ counts the number of excitons present in the state $|\nu\rangle$. The coupling constants $\gamma_{\mathbf{q}}$ are chosen specific for the deformation potential coupling to longitudinal acoustic phonons, which has been shown to be dominant for typical self-assembled GaAs QDs 16.37. As described in more detail in Ref. 37, the coupling constants $\gamma_{\mathbf{q}}$ depend on the electronic wave functions $\Psi_{e(h)}$ as well as on the deformation potential constants $D_{e(h)}$ for electrons (e) and holes (h), respectively. For simplicity, we assume the wave functions to be the ground-state solutions of a spherically symmetric harmonic potential, i.e., $\Psi_{e(h)}(\mathbf{r}) \propto \exp \left(-r^{2} /\left(2 a_{e(h)}^{2}\right)\right)$, and refer to $a_{e}$ as the QD radius. The characteristics of the exciton-phonon coupling can be expressed by the spectral density $J(\omega)=\sum_{\mathbf{q}}\left|\gamma_{\mathbf{q}}\right|^{2} \delta\left(\omega-\omega_{\mathbf{q}}\right)$, which under the above assumptions reads 37

$$
J(\omega)=\frac{\omega^{3}}{4 \pi^{2} \rho \hbar c_{s}^{5}}\left[D_{e} e^{-\omega^{2} a_{e}^{2} /\left(4 c_{s}^{2}\right)}-D_{h} e^{-\omega^{2} a_{h}^{2} /\left(4 c_{s}^{2}\right)}\right]^{2}
$$

with $\rho$ being the mass density. For our present calculations we use material parameters specific for GaAs taken from the literature $\frac{38}{-} \rho=5370 \mathrm{~kg} / \mathrm{m}^{3}, c_{s}=5110 \mathrm{~m} / \mathrm{s}$, $D_{e}=7.0 \mathrm{eV}$, and $D_{h}=-3.5 \mathrm{eV}$. For the QD size we choose $a_{e}=3 \mathrm{~nm}$ and set $a_{e} / a_{h}=1.15$ assuming equal potentials but taking into account the different effective masses of electrons and holes for GaAs. The spectral density for these parameters is shown in Fig. 1(c) where it can be seen that $J(\omega)$ exposes a clear maximum at $\hbar \omega=E_{J}^{\max } \approx 2 \mathrm{meV}$. The electron-phonon interaction leads to a polaron-shift $\delta_{p h}$ of the exciton and biexciton energy, such that when driving the exciton-to-ground state transition the resonant excitation is on the shifted exciton energy $\hbar \tilde{\omega}_{X}=\hbar \omega_{X}-\delta_{p h}$. In this paper we are mostly interested in detuned excitations and define the detuning as the difference between the laser energy and 
a)

b) $\Delta_{B} \underline{\hat{\imath} \cdots} \mid B>$

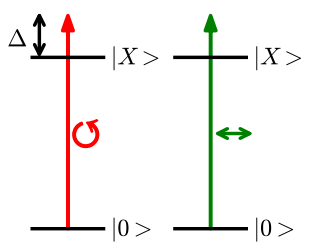

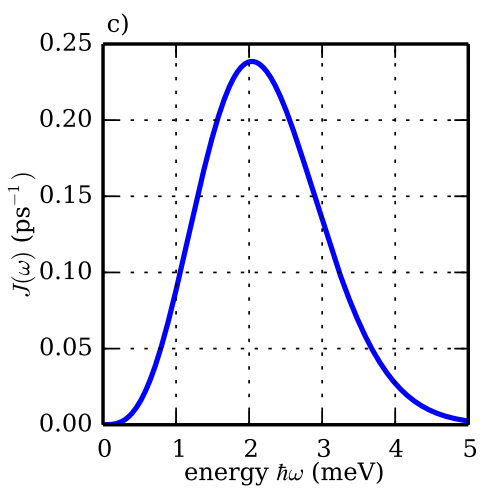

FIG. 1. (Color online) Energetic level diagram of (a) the exciton system with circular polarized excitation and (b) the exciton-biexciton system with linear polarized excitation. (c) Spectral density of the phonon coupling as a function of energy for the parameters used in our simulations (see text).

the polaron-shifted exciton energy, i.e.,

$$
\Delta=\hbar \omega_{L}-\hbar \widetilde{\omega}_{X}
$$

To study the time evolution of the electronic QD occupations under excitation with the laser field we employ an implementation of a real-time path-integral approach ${ }^{39}$. This method allows a numerically exact treatment of the above model despite the infinite number of LA phonon modes and yields the dynamics of the reduced electronic density matrix of the QD including arbitrary multi-phonon processes and taking into account all nonMarkovian effects. We assume the QD to be initially in a product state of the electronic ground-state and a thermal equilibrium of the phonon modes at temperature $T=1 \mathrm{~K}$.

\section{RESULTS}

We start by analyzing the phonon-induced relaxation using continuous excitation that is switched on instantaneously. Then we will apply short pulses with different pulse shapes to analyze the influence of the adiabaticity of the dressing and undressing process in view of high-fidelity state preparation. For this analysis we will also visualize the system trajectory on the Bloch sphere. Finally, we will demonstrate the selective addressing of all three states in the exciton-biexciton system using the phonon-assisted state preparation protocol.

\section{A. Phonon-induced relaxation}

The phonon-induced relaxation can be best analyzed by considering a continuous excitation of the QD with a constant field strength. Further, the laser field shall be circularly polarized such that our model can be restricted
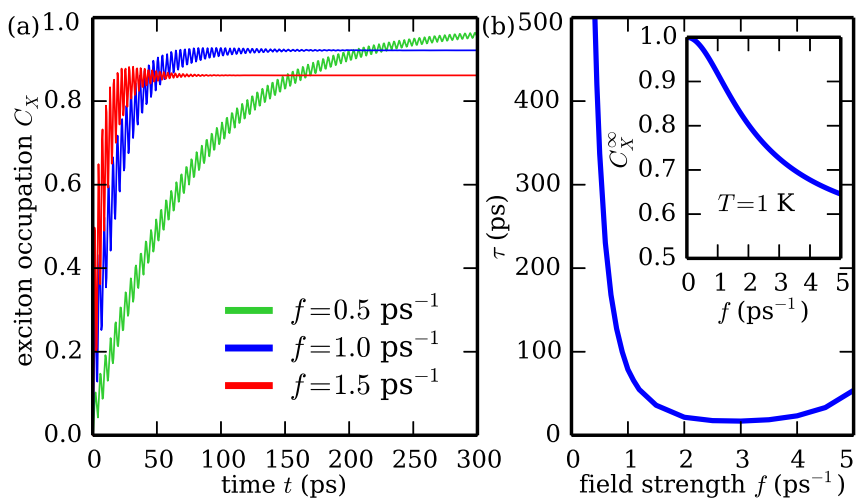

FIG. 2. (Color online) (a) Exciton occupation $C_{X}$ as a function of time for different field strengths $f=0.5 \mathrm{ps}^{-1}, 1.0 \mathrm{ps}^{-1}$ and $1.5 \mathrm{ps}^{-1}$. (b) Time $\tau$ after which the time average of the oscillations of $C_{X}$ has reached $99 \%$ of $C_{X}^{\infty}$ (see text) as a function of the field strength $f$. Inset of $(\mathrm{b}): C_{X}^{\infty}$ as a function of the field strength $f$. The detuning is $\Delta=1.0 \mathrm{meV}$.

to two electronic levels, as we explained in the previous section. Generally, for a weak coupling between the electronic states and the phonon environment the standard expectation for the driven QD dynamics is that the Markovian approximation is well justified and that in the long-time limit the relaxation leads to a thermal occupation of the dot-photon dressed states ${ }^{33,40}$ [c.f. Eq. (3)]. In a very good approximation, this has been shown to hold also true for the two-level model of the QD with standard GaAs-type parameters considered here ${ }^{26}$. For very low temperatures, the system ends up mainly in the lower dressed state $\left|\psi_{\text {low }}\right\rangle$, which corresponds to an exciton occupation of

$$
C_{X}^{\infty} \approx \cos ^{2}(\Theta)=\frac{1}{2}\left(1+\frac{\Delta}{\sqrt{\hbar^{2} f^{2}+\Delta^{2}}}\right) .
$$

Figure 2(a) shows the simulated temporal evolution of the exciton state under constant excitation for a detuning of $\Delta=1.0 \mathrm{meV}$ and three different field strengths $f$. Here, the laser field is switched on instantaneously at $t=0$ ps. The occupations perform damped Rabi oscillations around a mean value that approaches a constant value. For a decreasing field strength the stationary exciton occupation rises as can also be seen in the inset of Fig. 2(b) where $C_{X}^{\infty}$ given by Eq. (9) is shown as a function of $f$ for $\Delta=1.0 \mathrm{meV}$. Most importantly for phononassisted exciton preparation, the stationary exciton occupation is very close to one and only limited by the finite temperature for sufficiently small field strengths. Larger values of $f$, however, strongly reduce $C_{X}^{\infty}$.

On the other hand, when we look at the time required to reach the final state, we find that for small field strengths $f$, the time to reach the final state becomes longer. This is quantified in Fig. 2(b), where we have plotted the time $\tau$ it takes for the mean value of the oscillations to reach $99 \%$ of the exciton occupation expected for a thermal distribution of the dot-photon 
dressed states $C_{X}^{\infty}$ as a function of the field strength. For example at $f=0.5 \mathrm{ps}^{-1}$ a relaxation time of several hundred ps is required, which might exceed the time until other relaxation processes occurring on longer time scales than the phonon-induced relaxation such as the radiative decay which is not considered here, come into play. Therefore such a low driving strength does not yield a sufficiently fast relaxation for phonon-assisted state preparation. Indeed, we find that for $f \rightarrow 0$ the time needed for the state preparation diverges.

To understand the varying relaxation times for different driving strengths one has to keep in mind that the pure dephasing type phonon coupling does not induce direct transitions between the electronic states, but the phonon-induced relaxation is only enabled by the laser field. Transitions between the QD states only take place due to a non-vanishing overlap of both of the photon dressed states with the exciton state, which in turn is coupled to the phonon-environment. For an efficient relaxation also the Rabi splitting $\Omega$, i.e. the difference between the two dressed state energies, needs to be close to typical phonon energies and ideally matches the maximum of the spectral density of the phonon coupling $J(\omega)$ [c.f. Eq. (8)]. Both of these properties of the relaxation rate are also captured by a simple application of Fermi's Golden Rule, which yields a relaxation time between the upper dressed state without phonons and the lower dressed state with one phonon of

$$
\tau_{\text {relax }}=\left(\frac{\pi}{2} \sin ^{2}(2 \Theta) J(\Omega)\right)^{-1}
$$

Here it can be seen that the mixing angle $\Theta$ as well as the spectral density evaluated at the Rabi splitting $J(\Omega)$ enter the relaxation rate. According to the simulations the maximal relaxation for the detuning $\Delta=1.0 \mathrm{meV}$ used here occurs for a field strength around $2.7 \mathrm{ps}^{-1}$, which is reflected by the position of the minimum of the time needed for an almost complete relaxation plotted in Fig. 2(b). In a good approximation this is expected from the rough estimation in Eq. (10). Despite the short relaxation time at the optimal field strength, which is below 20 ps, such a strong driving field is not applicable for high-fidelity state preparation either, because the achieved final occupation of below 0.8 is too far from the desired fidelity of one.

Looking at our results, it becomes clear that the maximum exciton occupation for a given detuning can only be realized for almost vanishing field strengths where on the other hand it takes arbitrarily long times to complete the relaxation process. Therefore, we conclude, that when only the phonon relaxation process of the preparation takes place, there is a trade-off between reaching the targeted state with high fidelity and realizing fast preparation times.
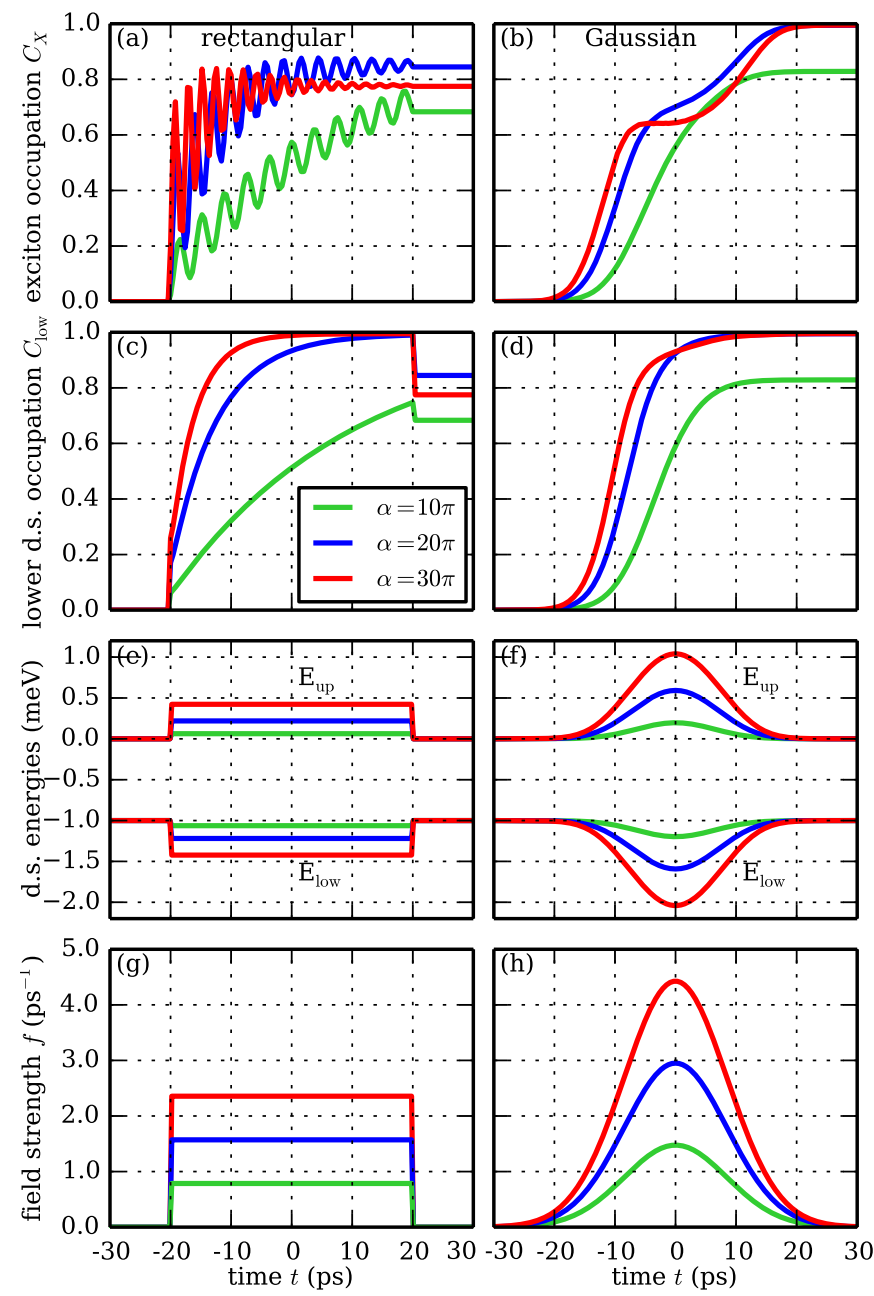

FIG. 3. (Color online) Dynamics for an off-resonant excitation of the QD with rectangular pulses (left) having a length of 40 ps and Gaussian pulses (right) with a FWHM of 20 ps. The pulse areas are $\alpha=10 \pi$ (green), 20 (blue) and $30 \pi$ (red). (a),(b) Exciton occupation $C_{X},(\mathrm{c}),(\mathrm{d})$ occupation of the energetically lower dressed state $C_{\text {low }}$, (e),(f) instantaneous energy of the upper and lower dressed state $E_{\text {up } / \text { low }}$, (g),(h) pulse envelopes. The detuning is $\Delta=1 \mathrm{meV}$.

\section{B. Dressing and Undressing}

In the previous section the switch-on of the laser field has been taken to be instantaneous. This implies that a sudden transformation of the photon dressed states occurs at the beginning of the pulse. Similarly, when switching off the laser field the photon dressed states are transformed back to the pure ground and exciton states. To understand the importance of these dressing and undressing processes in terms of fast and high fidelity phonon-assisted state preparation, we now look at excitations with different pulse shapes. First we compare an excitation of the two-level system with rectangular and Gaussian pulses. Later, we approximate the Gaussian pulse using a rectangular pulse with softened edges. 
Fig. 3 shows the temporal evolution under excitation for the rectangular (left) and Gaussian (right) pulses including (a),(b) the exciton occupation, (c),(d) the lower dressed state occupation, (e),(f) the instantaneous dressed state energies and $(\mathrm{g}),(\mathrm{h})$ the pulse envelopes of the electric field. The length of the rectangular pulses is chosen as $40 \mathrm{ps}$, which is twice the full width of half maximum (FWHM) of the Gaussian pulses, which is set to 20 ps. All calculations are performed for a detuning of $\Delta=1.0 \mathrm{meV}$ and for three different pulse areas $\alpha=\int_{-\infty}^{\infty} f(t) \mathrm{dt}$ with $\alpha=10 \pi$ (green curves), $20 \pi$ (blue curves), and $30 \pi$ (red curves).

Let us start with the rectangular pulse (left panels in Fig. 3). The laser pulse sets in instantaneously at $t=-20 \mathrm{ps}$ and besides damped Rabi oscillations there is an overall increase of the exciton occupation that depends on the strength of the pulse [Fig. 3) (a) and see also Fig. 2(a)]. In the dressed state picture this behavior can be understood as follows: When there is no laser pulse, the dressed states are equal to the bare states where for a positive detuning the ground state corresponds to the upper dressed state $E_{\text {up }}$ and the exciton state corresponds to the lower dressed state $E_{\text {low }}$. Note that in this picture the energies of the photons needed for the excitation are counted as part of the dressed state energies. As soon as the laser pulse sets in the dressed states become a mixture of ground and exciton state [cf. Eq. (3)] with shifted energies shown in Fig. 3(e), i.e. the bare QD states become dressed by the laser field. Due to their overlap with the ground-state both dressed states instantly become occupied, which is reflected by Rabi oscillations in the bare states $^{28}$. Phonons induce transitions between the dressed states 11,20 and at low temperatures transitions to the lower dressed state, that correspond to phonon emission, outweigh. Therefore, during the pulse the lower dressed state becomes more and more occupied as it can be seen in Fig. 3(c). This also means that the exciton occupation successively approaches its stationary value $C_{X}^{\infty}$, which depends on the exciton contribution to the lower dressed state but is above 0.5 for all field strengths and positive detunings [c.f. Eq. (9)]. When the laser pulse is switched off at $t=40$ ps the exciton occupation [Fig. 3)(a)] keeps the value it has right before the switch-off. At the same time the dressed states are abruptly transformed back to the bare QD states due to the sudden stop of the rectangular pulse, which means that the undressing takes place instantaneously. This is reflected by a step-like drop of the occupation of the lower dressed state that can be seen in Fig. 3](c). For the weakest pulse $(\alpha=10 \pi)$ the final state is not reached within the time window of $40 \mathrm{ps}$, while for the higher pulse areas the relaxation process is mostly completed. Either way, the final exciton occupation stays below 0.9 for all of the three rectangular pulses and in fact no matter which rectangular shape is assumed for pulse lengths of a few tens of ps one never achieves a final value close to one due to the trade-off situation described in the previous section.

The situation is quite different for the Gaussian pulses shown in the right column of Fig. 3. In this case, no oscillations of the exciton occupation are visible and instead $C_{X}$ smoothly rises to its final value that for pulse areas $\alpha=20 \pi$ and $30 \pi$ is considerably higher than for the rectangular pulses and practically reaches 1.0 as it can be seen in Fig. 3(b). Like for the rectangular pulses, a phonon relaxation process takes place from the upper to the lower dressed state yielding a steady increase of the occupation of the lower dressed state in Fig. 3(d). However, because the field strength for Gaussian pulses is time-dependent, the bare QD state contributions of the dressed states change during the pulse. This is also reflected by the time dependence of the dressed state energies shown in Fig. 3(f), which can be used to extract the Rabi splitting at a given time. For the weakest Gaussian pulse shown here $(\alpha=10 \pi)$, and similar to the case of the weakest rectangular pulse, the phonon-induced relaxation is too weak to complete the relaxation process within the duration of the pulse. This is because the Rabi splitting is well below the maximum of the phonon density at energy $E_{J}^{\max } \approx 2 \mathrm{meV}$ [c.f. Fig. 1(c)] even at the maximum of the pulse where the Rabi splitting reaches its maximum value of approx. $1.4 \mathrm{meV}$ [c.f. Fig. 3(f)]. In contrast, for the higher pulse areas $\alpha=20 \pi$ and $\alpha=30 \pi$ the relaxation is more effective and leads to a full occupation of the lower dressed state. At the pulse maximum the Rabi splitting for the $30 \pi$ pulse already becomes larger $(3.0 \mathrm{meV})$ than $E_{J}^{\max }$ temporarily leading to a weakening of the relaxation which is visible by a reduced gain of the lower dressed state occupation [red curve in Fig. 3(d)] around $t=0$ ps. Most interestingly, the phonon-induced relaxation is practically complete around $t=10 \mathrm{ps}$ while the exciton occupation obtained by the stronger pulses is still far from its final value and therefore the remaining increase of $C_{X}$ cannot be attributed to the relaxation. The final value of $C_{X}$ is only reached within a second phase of increase that takes place while the pulse is switched-off. This is only possible because during the switch-off the dressed states are adiabatically undressed, i.e. adiabatically transformed back to the bare QD states. In this process the ground state component of the then almost fully occupied lower dressed state is reduced to zero which yields a drastic increase of the exciton occupation. Importantly, a necessary precondition for this increase is that the undressing takes place slow enough such that the system can follow the transformation of the dressed states adiabatically and the occupation stays in the lower dressed state. For example this is not fulfilled for the instantaneous undressing in case of the rectangular pulses discussed earlier where the lower dressed state occupation experiences a step-like drop at the end of the pulse [Fig. 3(c)] that does not occur for Gaussian pulses [Fig. 33(d)].

As it turns out the adiabatic undressing towards the end of the pulse is in fact essential for a successful fast exciton preparation. The absence of the adiabatic ending prevents the phonon-assisted state preparation protocol from working with high fidelity for short rectangular 


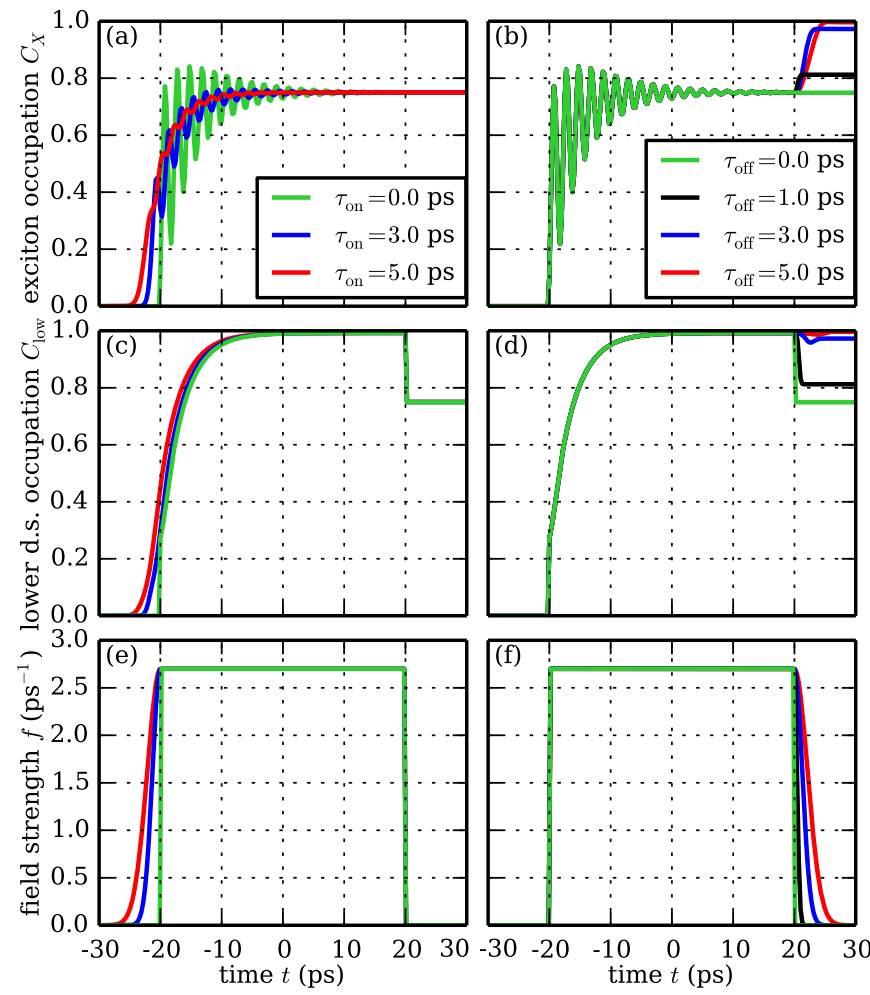

FIG. 4. (Color online) Time dependent exciton occupation $C_{X}$ under pulsed excitation detuned by $\Delta=1.0 \mathrm{meV}$ for different (a) switch-on (b) and switch-off times (see key). (c),(d) corresponding occupations of the energetically lower dressed state $C_{\text {low }}$ and (e), (f) corresponding pulse envelopes.

pulses and any other pulse shapes with too fast switch-off times.

Having seen that the temporal evolution of the dressing and undressing plays a crucial role, we investigate in more detail the role of the switch-on and switch-off phase. To this end we choose a special pulse shape that is designed to highlight the important role of these two processes on the preparation process. The thermalization of the dressed states is obviously achieved best by a constant part of the pulse that is chosen such that the coupling to the phonon environment is maximal ( $f=2.7 \mathrm{ps}^{-1}$ for the parameters chosen here) and that is sufficiently long to complete the thermalization. In our case, the duration of this part of constant driving is chosen to be 40 ps. In order to systematically study the influences of the switchon (switch-off) characteristics we add the left (right) half of a Gaussian pulse with FWHM $\tau_{\text {on }}\left(\tau_{\text {off }}\right)$ before (after) the constant part of the pulse envelope as illustrated in Fig. 4(e)[(f)]. Here, we compare a strictly rectangular pulse with $\tau_{\text {on } / \text { off }}=0$ ps (green) with pulses of different switch-on/off times $\tau_{\text {on/off }}=1$ ps (black), 3 ps (blue) and 5 ps (red). The first row [panels (a) and (b)] shows the corresponding evolution of $C_{X}$, while the lower dressed state occupation is plotted in the second row [panels (c) and (d)] and the pulse envelopes are shown in the third row [panels (e) and (f)].
Looking at the left panels of Fig. 4, we find that a longer switch-on time does not alter the resulting exciton occupation after the pulse, which is about 0.75 for all three cases. However, we find a significant reduction of the Rabi oscillations, which almost disappear for $\tau_{\text {on }}=5$ ps. The insensitivity of the final occupation with respect to the switch-on dynamics is related to the irreversible nature of the relaxation process which, in case of a complete relaxation, leads to a complete loss of memory of the initial state. We thus find that the first phase, i.e., the dressing, is necessary to start the relaxation process, but for a sufficiently long relaxation phase the details of the switch-on are irrelevant for the final fidelity.

In contrast, as demonstrated in the right panels of Fig. 4, a slower switch-off gives rise to a further gain of exciton occupation when the field strength is reduced to zero. This effect is considerably less pronounced if the switch-off time becomes too short and does not occur at all if the pulse stops instantaneously. For example for $\tau_{\text {off }}=5$ ps (red curve) $C_{X}$ increases by more than 0.2 during the switch-off, while for $\tau_{\text {off }}=1$ ps (black) the increase is below 0.05 . Thus, we conclude, that to yield a high fidelity state preparation the transformation of the dressed states into the bare QD states needs to happen slow enough in order to allow for an adiabatic evolution, i.e., the undressing needs to be adiabatic.

To sum up, for an efficient and fast phonon-assisted state preparation there are basically two features of the pulse envelope that are significant: First, there has to be a phase of the pulse where the QD thermalizes in the dressed state basis, which must be long enough to complete the relaxation. This phase can be chosen the shorter the stronger the phonon-induced relaxation is and therefore can be minimized when the field strength is such that the strength of the phonon-coupling is maximal. Therefore in this phase a constant field strength corresponding to the maximal relaxation rate is optimal to achieve a complete thermalization with a minimal pulse length. Second, the pulse must be switched-off slowly enough to allow the QD system for an adiabatic undressing of the states.

Although a Gaussian pulse shape does not meet these requirements in an optimal fashion it still works surprisingly well, mostly because the optimum for the ideal field strength at which the dressed state relaxation is maximal is not a sharp maximum, but rather a broad peak as it can be seen from Fig. 1] Therefore, even though a Gaussian pulse does not have a constant plateau, a sufficient relaxation takes place during most of the time of the pulse provided a reasonable value of the pulse area is chosen. Most importantly, deviations from the Gaussian pulse shape that go towards faster switch-off times, as they might be induced by a pulse-shaping setup, can be harmful to the efficiency of the phonon-assisted preparation protocol. 

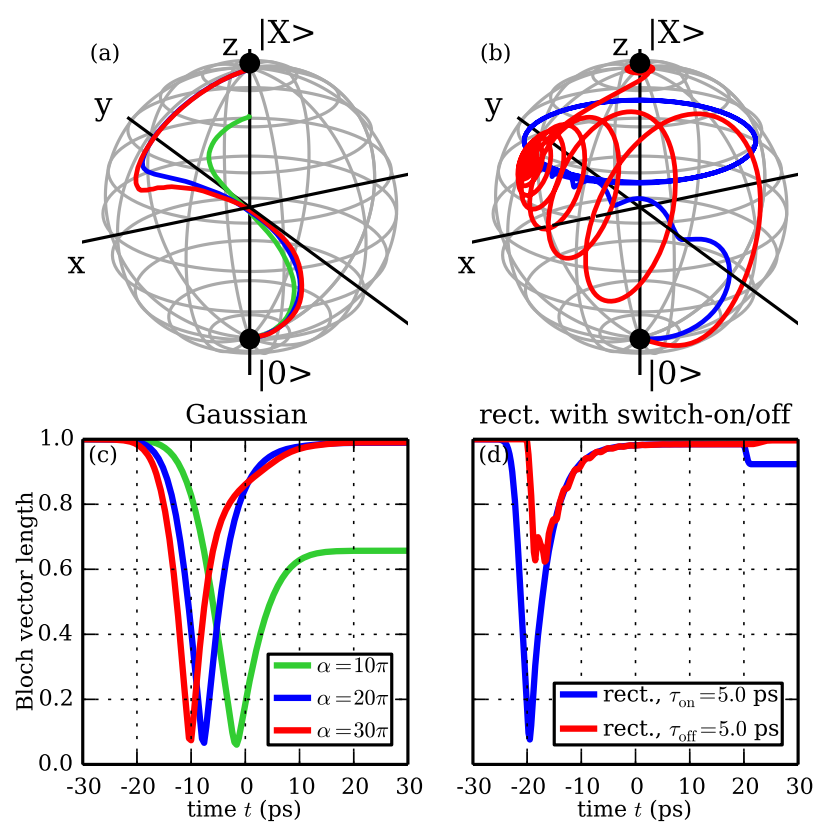

FIG. 5. (Color online) (a),(b) Illustration of the system trajectory on the Bloch sphere and (c),(d) length of the Bloch vector as a function of time for (a),(c) a Gaussian excitation with pulse areas $\alpha=10 \pi$ (green), $20 \pi$ (blue) and $30 \pi$ (red) and (b),(d) a rectangular excitation with different switchon/off times $\tau_{\text {on }}=5 \mathrm{ps} ; \tau_{\text {off }}=0$ ps (blue) and $\tau_{\text {on }}=0 \mathrm{ps;}$ $\tau_{\text {off }}=5 \mathrm{ps}$ (red). The detuning is $\Delta=1 \mathrm{meV}$.

\section{Interpretation on the Bloch sphere}

Another interesting aspect not highlighted so far is that the incoherent phonon scattering can result in a pure state, which even can be transformed to a bare QD state. This is best illustrated using the Bloch vector picture 41 . In this picture the projection of the Bloch vector on the $z$ axis represents the inversion, i.e., the difference between the occupations of the upper and the lower level of the two-level system, while the in-plane component reflects the polarization. Resonant lossless driving of a two-level system is reflected by the Bloch vector moving at the surface of the Bloch sphere taking the shortest path from one pole to the other corresponding to the well known coherent Rabi oscillations. Preparing the exciton state using a $\pi$ pulse then means going from the lower pole corresponding to the ground level to the upper pole corresponding to the excited level. A detuned excitation leads to a tilted oscillation, which starting from the lower pole does not reach the upper pole. If the system is fully coherent, the Bloch vector stays on the surface, i.e., its length is constantly one. If decoherence takes place, the length of the Bloch vector is decreased. Clearly, the phonon-assisted preparation involves an incoherent phonon-induced relaxation, but as we have seen it can still eventually lead to an almost perfect exciton state.

To analyze this in more detail we have calculated the trajectory of the Bloch vector of the driven QD system, which is shown in Fig. [5(a), (b) alongside the time evolution of the length of the Bloch vector presented in Fig. 5 (c),(d) for an excitation with Gaussian pulses (left panel) and for rectangular pulses with softened switch-on or switch-off (right panel). The corresponding pulse shapes can be seen in Fig. 3 (h) and Fig. 4 (e), (f).

Let us first look at the Gaussian pulses in the left column. When the pulse is switched on the Bloch vector leaves the surface of the sphere for all pulse areas in Fig. 5(a) $[\alpha=10 \pi$ (green), $\alpha=20 \pi$ (blue) and $\alpha=30 \pi$ (red)]. The corresponding vector length shown in Fig. 5(c) decreases as it is expected as the result of the pure-dephasing phonon coupling. Indeed, the loss of coherence turns out to be as high as $95 \%$. The time to reach the minimal Bloch vector length depends on the pulse area and is shorter for the stronger pulses where the relaxation is more efficient. The vector length would vanish at the minimum in the ideal case of a completely adiabatic dressing process and provided that the phonons realize a fully incoherent occupation transfer between the dressed states. In that case the electronic density matrix would stay diagonal in the dressed state basis all the time and coherences between the dressed states would neither build up due to the laser driving nor due to the phonons. The continuous occupation transfer from the upper to the lower dressed state would then necessarily lead to a zero of the Bloch vector length at some point in time. In our case, however, the minimal Bloch vector length has a small but finite value because the Gaussian pulses used in the simulations already induce some small coherences between the dressed states (not shown). Subsequently, the coherence between $|0\rangle$ and $|X\rangle$ is restored to a large degree and the Bloch vector length approaches 1 for the two stronger pulses as the phonon-assisted transitions result in an almost complete occupation of the lower dressed state which is again a pure state lying on the surface of the Bloch sphere. For the weaker pulse, $\alpha=10 \pi$ (green), the trajectory ends inside the Bloch sphere and the vector length stays well below 0.5 , because due to the insufficient pulse strength the relaxation does not complete. For the stronger pulses, the dressed states transform from a superposition of ground and exciton state into the pure exciton state during the adiabatic undressing, which corresponds to a motion of the Bloch vector on the surface towards the upper pole. It thus becomes obvious that the laser-driven QD evolution describes a way through the Bloch sphere to the upper pole, which is very different to the motion along the surface in the case of an inversion yielded by applying a resonant $\pi$ pulse.

A similar analysis can be done for the Bloch vector trajectory for rectangular pulses where the switchon or the switch-off edge is softened. The red curve in Fig. [5(b),(d) has a sharp switch-on and a smooth switchoff with $\tau_{\text {off }}=5 \mathrm{ps}$, while the blue curve corresponds to a smooth switch-on with $\tau_{\text {on }}=5$ ps and a sharp switch-off. When the switch-on is instantaneous (red curve), we see that the Bloch vector trajectory exhibits a spiral move- 

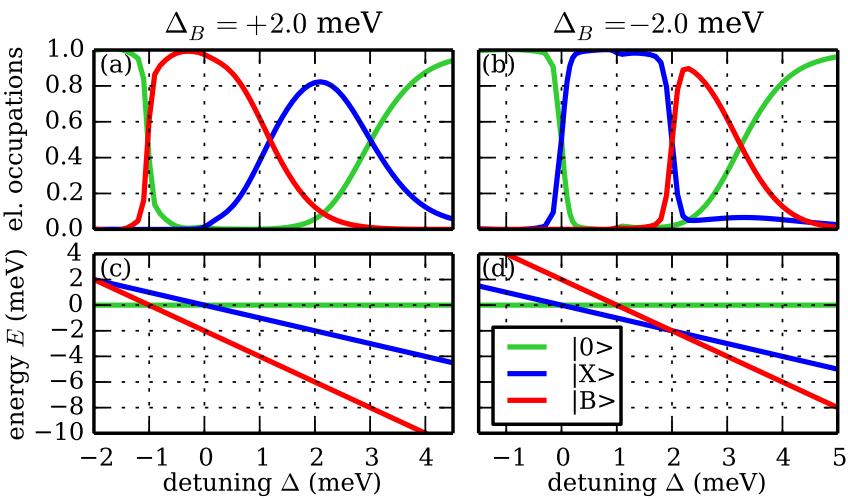

FIG. 6. (Color online) Occupations of the electronic levels $|0\rangle$ (green), $|X\rangle$ (blue) and $|B\rangle$ (red) after optically exciting the exciton-biexciton system with a Gaussian pulse of pulse area $\alpha=20 \pi$ and $\mathrm{FWHM}=20$ ps as a function of the detuning $\Delta$ for a (a) positive biexciton binding energy $\Delta_{B}=2.0 \mathrm{meV}$ and (b) negative biexciton binding energy $\Delta_{B}=-2.0 \mathrm{meV}$. (c) and (d) : corresponding energies of the QD states in the rotating frame.

ment within the Bloch sphere reflecting the damped Rabi oscillations. In agreement with the essentially complete relaxation, the spiral ends up on a point close to the surface of the Bloch sphere. For $\tau_{\mathrm{on}}=5$ ps (blue curve), the oscillations are less pronounced and the motion goes roughly along the axis of the spiral movement. The spiraling will eventually vanish for even larger values of $\tau_{\mathrm{on}}$, but the end point after the relaxation will be the same. If the laser pulse is switched off rapidly (blue curve), the z-component of the Bloch vector stays constant after the end of the pulse and performs a circular motion. In contrast, for $\tau_{\text {off }}=5 \mathrm{ps}$ an adiabatic undressing takes place during the switch-off and the Bloch vector approaches the upper pole.

\section{Selective state preparation in the exciton-biexciton system}

The clear understanding of the different phases of the phonon-assisted relaxation mechanism worked out so far turns out to be highly valuable to predict in an easy way the QD state that can be obtained by pulsed offresonant excitation for arbitrary initial states and also for the exciton-biexciton system illustrated in Fig. 1(b). To this end it is required as before that during the switchoff phase of the pulse the system evolves adiabatically. When the pulse duration is sufficiently long such that the relaxation all the way to the lowest dressed state is fully completed the prepared state at the end of the pulse is determined exclusively by the energetic order of the dressed states in the limit of vanishing pulse strength. Recalling that the dressed states are defined with respect to the rotating frame, the prepared state can technically be determined by subtracting from the energies of the QD states without light coupling the energy of the cor- responding number of photons needed for reaching that state and looking at the resulting order of the states. More specifically, zero photons have to be subtracted for the ground state, one photon for an exciton state and two photons for the biexciton state. The resulting state lowest in energy will be occupied predominantly at the end of the preparation process.

For example in the two-level system subtracting the laser energy from the laser-free QD state energies yields dressed state energies that are in the limit of vanishing field strength separated by the detuning between the laser and the polaron-shifted QD transition. For a positive detuning the energy of the exciton-like dressed state will be below the corresponding ground state-like dressed state energy in the rotating frame and consequently the phonon-assisted preparation protocol with adiabatic undressing prepares the exciton state. On the other hand, a negative detuning reverses the order of the dressed state energies and at low temperatures where there is mostly phonon emission the protocol prepares the QD ground state independent of the initial state $\underline{29}$.

In the case of an exciton-biexciton system subtracting the energy of the corresponding photons gives the energies of the QD states in the frame rotating with the laser frequency as $E_{0}^{\prime}=0, E_{X}^{\prime}=-\Delta, E_{B}^{\prime}=-\Delta_{B}-2 \Delta$. Thus, the energetic ordering depends also on the biexciton binding energy and we will consider both cases with positive and negative biexciton binding energy, which can both be realized depending on the QD geometry ${ }^{42}$. In the following we will refer to the dressed state that in the limit of vanishing field strength transforms into the $|0\rangle[|X\rangle$, $|B\rangle]$ state as $\left|0^{\prime}\right\rangle\left[\left|X^{\prime}\right\rangle,\left|B^{\prime}\right\rangle\right]$.

Let us first discuss the most commonly encountered situation of a QD with a positive biexciton binding energy $\Delta_{B}=2 \mathrm{meV}$. Figure [6 (a) shows the final occupation after excitation with a detuned Gaussian pulse with a FWHM of 20 ps and a pulse area of $\alpha=20 \pi$ as a function of the detuning, while Fig. 6 (c) shows the corresponding energies in the rotating frame. It can be seen that for detunings below the two-photon resonance, i.e., $\Delta<-\Delta_{B} / 2=-1 \mathrm{meV}$, the occupation remains in the ground state (green curve). This is consistent with the energetic order of the states, since $E_{0}^{\prime}$ is the lowest energy in this parameter region. At $\Delta=-1 \mathrm{meV}$ the energetic order of the dressed states changes, because for detunings above the two-photon resonance $E_{B}^{\prime}$ is the lowest energy. This leads to a significant drop of the final ground state occupation in favor of the biexciton occupation (red curve), which approaches its maximum close to one at $\Delta \approx-0.5 \mathrm{meV}$. At the one-photon resonance at $\Delta=0$, the energetic order changes once again and for all positive detunings $\left|0^{\prime}\right\rangle$ is the highest energy dressed state, while $E_{B}^{\prime}$ remains being the lowest energy. For sufficiently long pulses all the occupation would, of course, end up in the lowest branch resulting in the preparation of the biexciton. However, for $\Delta>1 \mathrm{meV}$ the energetic splitting $E_{X}^{\prime}-E_{B}^{\prime}>3 \mathrm{meV}$ exceeds the maximum of the phonon spectral density $E_{J}^{\max }$ lying around $2 \mathrm{meV}$ by 
far, which results in a very inefficient relaxation to the $\left|B^{\prime}\right\rangle$ state, which is not completed in the time window set by the pulse length. Therefore, instead, we observe a gain of the exciton occupation (blue curve) as soon as $E_{X}^{\prime}$ crosses $E_{0}^{\prime}$. The maximal exciton occupation of about 0.8 is reached around $\Delta=2 \mathrm{meV}$, where the energy splitting $E_{0}^{\prime}-E_{X}^{\prime}=2 \mathrm{meV}$ agrees with $E_{J}^{\max }$. Therefore, it turns out that for a system with more than two states like the one considered here an incomplete relaxation can also be advantageous for preparation purposes if a preparation of a QD state that in the rotating frame is not the lowest lying state, like in our case the exciton, is intended. An even higher exciton occupation is possible for a larger biexciton binding energy which favors transitions to $\left|X^{\prime}\right\rangle$, because the coupling to the lowest dressed state $\left|B^{\prime}\right\rangle$ in this case gets even more out of resonance. It also follows that whether the prepared state is the exciton or the biexciton can in principle be selected by suitably adjusting the pulse length for all positive detunings, because the final state depends on whether the relaxation completes during the pulse or whether the intermediate level corresponding to the exciton is still predominantly occupied when the pulse is switched off. Detunings higher than $2.0 \mathrm{meV}$ lead to a decrease of both the final exciton and the biexciton occupations, because the phonon-induced relaxation becomes weaker and weaker as the phonon environment becomes more and more out of resonance. Finally, for very large detunings above $\Delta=4.0 \mathrm{meV}$ the energetic splittings between the dressed states are so large that for the given pulse length practically no relaxation takes place and the QD remains in the ground-state.

The case of a negative biexciton binding energy of $\Delta_{B}=-2 \mathrm{meV}$ is shown in the right column. Fig. 6(b) shows the final occupations and the corresponding energies are plotted in Fig. 6(d). Similar to the case of positive biexciton binding energies the system remains in the ground state up to a detuning of 0 , since $E_{0}^{\prime}$ is the lowest energy. Between $\Delta=0$ and $2 \mathrm{meV}\left|X^{\prime}\right\rangle$ is the dressed state with the lowest energy. This energetic order appears exclusively for negative biexciton binding energies, resulting in a broad region where a complete preparation of the exciton occurs. This finding is somewhat surprising, because the two-photon resonance at $\Delta=1 \mathrm{meV}$ also lies within this interval, where an efficient preparation of the exciton state might be unexpected, but can easily be understood in the context described here. At $\Delta=2 \mathrm{meV}$ the energetically lowest state changes a second time and a sharp transition of the prepared state from the exciton to the biexciton occurs. However, for $\Delta>2.5 \mathrm{meV}$ the energy splittings between the dressed states that are connected to the excitonic states and $\left|0^{\prime}\right\rangle$ already become too large for a complete thermalization to take place during the length of the pulse. This leads to a significant reduction of the biexciton occupation for higher detunings and above $\Delta=5 \mathrm{meV}$ the QD does not get affected by the pulse anymore staying in the groundstate. Importantly, also in the case of a negative biexci- ton binding energy the targeted state can be derived from the energetic order shown in Fig. 6(d), demonstrating the correctness of the description found for the dynamics of the phonon-induced preparation process also in the case of the exciton-biexciton system.

As we have seen, to selectively address a state in an exciton-biexciton system by phonon-assisted state preparation the biexciton binding energy needs to be considered carefully, while the detuning $\Delta$ and the pulse length can act as control parameters to choose the targeted state.

\section{CONCLUSIONS}

We have analyzed the dynamics of an optically driven semiconductor QD coupled to longitudinal acoustic phonons for different off-resonant excitation conditions focusing on two main questions: a) what are the requirements for a fast state preparation using off-resonant driving and b) how is the prepared state selected. We demonstrated that a fast high fidelity preparation process not only relies on an efficient phonon-induced relaxation between the dot-photon dressed states, but also on a successful adiabatic undressing of the states. To understand the influence of the adiabatic undressing we compared the exciton occupations produced by Gaussian and rectangular pulse shapes and systematically studied the influence of the switch-on and switch-off times. This analysis revealed that while the switch-on time plays a subordinate role it is crucial that the pulse is switched off slowly enough. Furthermore, we analyzed the coherence properties during the state preparation process and revealed that the incoherent phonon scattering can also restore coherence and the system trajectory takes a way through the Bloch sphere to the opposite pole. Finally, we showed that the concept of phonon-assisted state preparation by adiabatic undressing also applies to the exciton-biexciton system, where an easy prediction of the prepared state is possible. When the pulse is long enough to support a full relaxation the prepared state is the one that is lowest in energy in the rotating frame in the limit of vanishing field strength. We demonstrated that even pulses too short for a full relaxation can be used for preparing intermediate states that are not the lowest in energy in the rotating frame. Altogether, it is demonstrated that in an exciton-biexciton system the decisive parameters for selecting the prepared state are the detuning, the biexciton binding energy and the pulse length.

\section{ACKNOWLEDGMENTS}

A.M.B. and V.M.A. gratefully acknowledge the financial support from Deutsche Forschungsgemeinschaft via the Project No. AX 17/7-1. D.E.R. is thankful for financial support from the German Academic Exchange Service (DAAD) within the P.R.I.M.E. programme. 
* andreas.barth@uni-bayreuth.de

1 D. Bouwmeester, A. K. Ekert, and A. Zeilinger, The Physics of Quantum Information (Springer, Berlin, 2000).

2 E. Biolatti, R. C. Iotti, P. Zanardi, and F. Rossi, Phys. Rev. Lett. 85, 5647 (2000).

${ }^{3}$ P. Chen, C. Piermarocchi, and L. J. Sham, Phys. Rev. Lett. 87, 067401 (2001).

4 X. Q. Li, Y. W. Wu, D. Steel, D. Gammon, T. H. Stievater, D. S. Katzer, D. Park, C. Piermarocchi, and L. J. Sham, Science 301, 809 (2003).

${ }^{5}$ C. Piermarocchi, P. Chen, Y. S. Dale, and L. J. Sham, Phys. Rev. B 65, 075307 (2002).

6 S. J. Boyle, A. J. Ramsay, F. Bello, H. Y. Liu, M. Hopkinson, A. M. Fox, and M. S. Skolnick, Phys. Rev. B 78, 075301 (2008).

7 M. A. Nielsen and I. Chuang, Quantum Computation and Quantum Information (Cambridge University Press, England, 2000).

8 E. Moreau, I. Robert, L. Manin, V. Thierry-Mieg, J. M. Gérard, and I. Abram, Phys. Rev. Lett. 87, 183601 (2001).

9 R. Stevenson, R. J. Young, P. Atkinson, K. Cooper, D. A. Ritchie, and A. J. Shields, Nature 439, 179 (2006).

10 M. Müller, S. Bounouar, K. D. Jöns, M. Glässl, and P. Michler, Nature Photonics 8, 224 (2014).

11 D. E. Reiter, T. Kuhn, M. Glässl, and V. M. Axt, J. Phys. Cond. Matter 26, 423203 (2014).

12 A. J. Ramsay, Semicond. Sci. Technol. 25, 103001 (2010).

13 A. Zrenner, E. Beham, S. Stufler, F. Findeis, M. Bichler, and G. Abstreiter, Nature 418, 612 (2002).

14 P. Machnikowski and L. Jacak, Phys. Rev. B 69, 193302 (2004).

15 A. Vagov, M. D. Croitoru, V. M. Axt, T. Kuhn, and F. M. Peeters, Phys. Rev. Lett. 98, 227403 (2007).

16 A. J. Ramsay, A. V. Gopal, E. M. Gauger, A. Nazir, B. W. Lovett, A. M. Fox, and M. S. Skolnick, Phys. Rev. Lett. 104, 017402 (2010).

17 E. R. Schmidgall, P. R. Eastham, and R. T. Phillips, Phys. Rev. B 81, 195306 (2010).

18 C. M. Simon, T. Belhadj, B. Chatel, T. Amand, P. Renucci, A. Lemaitre, O. Krebs, P. A. Dalgarno, R. J. Warburton, X. Marie, and B. Urbaszek, Phys. Rev. Lett. 106, 166801 (2011).

19 Y.-J. Wei, Y.-M. He, M.-C. Chen, Y.-N. Hu, Y. He, D. Wu, C. Schneider, M. Kamp, S. Höfling, C.-Y. Lu, and J.-W. Pan, Nano Letters 14, 6515 (2014).

20 S. Lüker, K. Gawarecki, D. E. Reiter, A. Grodecka-Grad, V. M. Axt, P. Machnikowski, and T. Kuhn, Phys. Rev. B 85, 121302 (2012).

21 M. Glässl, A. M. Barth, K. Gawarecki, P. Machnikowski, M. D. Croitoru, S. Lüker, D. E. Reiter, T. Kuhn, and
V. M. Axt, Phys. Rev. B 87, 085303 (2013)

${ }^{22}$ K. Gawarecki, S. Lüker, D. E. Reiter, T. Kuhn, M. Glässl, V. M. Axt, A. Grodecka-Grad, and P. Machnikowski, Phys. Rev. B 86, 235301 (2012)

23 A. Debnath, C. Meier, B. Chatel, and T. Amand, Phys. Rev. B 86, 161304 (2012).

24 A. Debnath, C. Meier, B. Chatel, and T. Amand, Phys. Rev. B 88, 201305 (2013).

25 R. Mathew, E. Dilcher, A. Gamouras, A. Ramachandran, H. Y. S. Yang, S. Freisem, D. Deppe, and K. C. Hall, Phys. Rev. B 90, 035316 (2014).

26 M. Glässl, A. Vagov, S. Lüker, D. E. Reiter, M. D. Croitoru, P. Machnikowski, V. M. Axt, and T. Kuhn, Phys. Rev. B 84, 195311 (2011).

27 D. E. Reiter, S. Lüker, K. Gawarecki, A. Grodecka-Grad, P. Machnikowski, V. M. Axt, and T. Kuhn, Acta Phys. Pol. A 122, 1065 (2012).

28 S. Hughes and H. J. Carmichael, New Journal of Physics 15, 053039 (2013)

29 M. Glässl, A. M. Barth, and V. M. Axt, Phys. Rev. Lett. 110, 147401 (2013).

30 P.-L. Ardelt, L. Hanschke, K. A. Fischer, K. Müller, A. Kleinkauf, M. Koller, A. Bechtold, T. Simmet, J. Wierzbowski, H. Riedl, G. Abstreiter, and J. J. Finley, Phys. Rev. B 90, 241404 (2014)

31 J. H. Quilter, A. J. Brash, F. Liu, M. Glässl, A. M. Barth, V. M. Axt, A. J. Ramsay, M. S. Skolnick, and A. M. Fox, Phys. Rev. Lett. 114, 137401 (2015).

32 S. Bounouar, M. Müller, A. M. Barth, M. Glässl, V. M. Axt, and P. Michler, Phys. Rev. B 91, 161302 (2015).

33 A. J. Leggett, S. Chakravarty, A. T. Dorsey, M. P. A. Fisher, A. Garg, and W. Zwerger, Rev. Mod. Phys. 59, 1 (1987).

34 H. Dekker, J. Phys. Cond. Matter 20, 3643 (1987).

35 W. Langbein, P. Borri, U. Woggon, V. Stavarache, D. Reuter, and A. D. Wieck, Phys. Rev. B 69, 161301 (2004).

36 D. J. Tannor, Introduction to quantum mechanics (University Science Books, Sausalito, California, 2007).

37 B. Krummheuer, V. M. Axt, and T. Kuhn, Phys. Rev. B 65, 195313 (2002).

38 B. Krummheuer, V. M. Axt, T. Kuhn, I. D'Amico, and F. Rossi, Phys. Rev. B 71, 235329 (2005).

39 A. Vagov, M. D. Croitoru, M. Glässl, V. M. Axt, and T. Kuhn, Phys. Rev. B 83, 094303 (2011).

40 U. Weiss, Quantum dissipative systems, 2nd ed. (World Scientific, Singapore, 1999).

41 L. Allen and J. H. Eberly, Optical Resonance and TwoLevel Atoms (John Wiley and Sons, New York, 1975).

42 P. Michler, Single Semiconductor Quantum Dots (Springer-Verlag, Berlin, 2009). 